DÉCOLONISATION ET CONSTRUCTION NATIONALE

AFRIQUE, ASIE ET QUÉBEC

Titre: SOCIALISME ET dÉCOLONISATION DANS LE QuÉBEC DE LA RÉVOLUTION TRANQUiLle À TRAVERS LA REVUE SOCIALISTE ET PARTI PRIS

Auteur(s): StÉPHANIE JodoIn, UniVERSITÉ DE SHERBROOKE

Publication: Décolonisation et construction nationale: Afrique, Asie et Québec

PAGE: 101-114.

Directeurs: PATrick Dramé, PAScal SCALlon-Chouinard et FrançoISE NozATI

ÉditeuR: LES ÉDITIONS DE L'UNIVERSITÉ DE SHERBROOKE, 2016.

ISBN: 978-2-7622-0351-6

URI: HTTP://HDL.HANDLE.NET/11143/8760

DOI: HTTP://DX.DOI.ORG/10.17118/11143/8760 


\section{Socialisme et décolonisation dans le Québec de la Révolu- tion tranquille à travers La Revue socialiste et Parti Pris}

Stéphanie Jodoin

La politique internationale des années 1960 voit s'imposer la question de la décolonisation des pays du Tiers-monde. Les mouvements nationalistes d'alors s'affirment avec force et imposent une pression qui, jumelée aux actions politiques internationales, conduit progressivement à l'indépendance de plusieurs territoires africains. De nombreux intellectuels originaires des pays en voie de décolonisation publient et se font entendre. Les grandes puissances tentent quant à elles d'attirer les nouveaux pays décolonisés dans leur sphère d'influence. Dans le contexte de la Guerre froide, l'alignement des pays décolonisés et le choix d'une idéologie deviennent d'ailleurs un enjeu d'envergure. Les luttes contre la colonisation et l'impérialisme, mais aussi contre le communisme ou le capitalisme, font partie des débats qui influencent inévitablement le rythme et la méthode de décolonisation.

À la même époque, au Québec, se multiplient les mouvements de revendication de toute sorte. L'indépendance, entre autres, devient une question de plus en plus discutée. Influencée par le contexte international, cette idée s'est progressivement développée en une idéologie originale parmi la gauche nationaliste, que Sean Mills nomme le « socialisme de décolonisation $»^{1}$.

Cette idéologie est diffusée à travers les principales revues de la gauche radicale québécoise qui sont, comme l'affirme Andrée Fortin, « des tremplins d'idées neuves et des lieux de débats². » Elle y connaît un développement accéléré dès la fin des années 1950 et jusqu'à la fin des années 1960, puis perd en popularité au début des années 1970. Deux revues se démarquent alors par leur importance : La Revue socialiste (1959-1960), qui relève de l'action de Raoul Roy - I'un des premiers penseurs du « socialisme de décolonisation »-, ainsi que la revue Parti pris (1963-1968) qui, souvent décrite comme l'héritière idéologique de La Revue socialiste, vient donner un visage nouveau, plus jeune et plus populaire, au mouvement de décolonisation. Or, bien que la filiation entre les deux revues soit indéniable, celles-ci diffèrent pourtant sur plusieurs points. En effet, le nationalisme de la revue de Roy, par exemple, se

1. Sean Mills, The Empire Within. Postcolonial Thought and Political Activism in Sixties Montreal, Montréal, McGillQueen's University Press, 2010, p. 4-11.

2. Andrée Fortin, «Les revues de gauche au Québec, 1960-1970 », Bulletin d'histoire politique, vol. 19, n² (2010), p. 78-86. 
rapproche beaucoup plus d'un «nationalisme traditionnel », alors que Parti pris lie plutôt le discours des décolonisations à une vision plus moderne de la nation ${ }^{3}$. Loin d'évoluer par elles-mêmes, les revues s'inspirent d'auteurs étrangers influents - tels Albert Memmi, Frantz Fanon, Aimé Césaire et Jacques Berque, à titre d'exemple ${ }^{4}$ qui placent au cœur de leurs écrits les luttes d'indépendance et l'affirmation des pays du Tiers-monde ${ }^{5}$.

L'analyse de ces deux revues permet de mieux comprendre le discours des décolonisations et son insertion progressive au Québec. Quels sont les arguments repris par les mouvements indépendantistes de gauche pour justifier la définition du Québec comme une colonie, et comment ceux-ci sont-ils développés? La période d'activité de La Revue socialiste et de Parti pris se suivant de près, il est possible d'observer, par leur comparaison, la complexité du discours qui, en fait, n'apparait ni uniforme, ni stable, et ce, de la fin des années 1950 jusqu'à la fin des années 1960. Durant cette période, les théories de la décolonisation sont en effet adoptées par les mouvements socialistes indépendantistes du Québec, mais évoluent aussi dans le sens d'une lutte contre l'impérialisme et le capitalisme. La gauche, quant à elle, se radicalise et se tourne davantage vers le marxisme-léninisme. Par le fait même, elle met un peu de côté le contexte québécois et l'indépendance ${ }^{6}$.

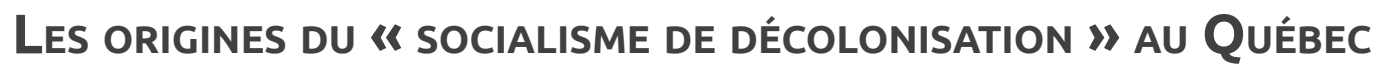

Au début des années 1960, alors que les notions de "progrès » et de "modernité » sont au cœur du développement social et économique de la province, les intellectuels québécois vont rapidement s'interroger sur les causes de la pauvreté plus marquée des Canadiens français et de leur « retard » en comparaison avec le reste de l'Occident ${ }^{7}$. Dans les faits, à I'heure de la prospérité, il est vrai que les Canadiens français, au Québec du moins, sont pour diverses raisons plus pauvres que leurs concitoyens canadiens-anglais ou que leurs voisins

3. Concernant le nationalisme québécois et son évolution, voir Louis Balthazar, Bilan du nationalisme au Québec, Montréal, L'Hexagone, 1986, 212 p.

4. Voir, notamment : Albert Memmi, Portrait du colonisé : précédé de portrait du colonisateur, Montréal, L'Étincelle,

1972 (1957), 146 p.; Frantz Fanon, Les damnés de la terre, Paris, La Découverte, coll. « La Découverte/Poche », no 134, 2002 (1961),

311 p.; Aimé Césaire, Discours sur le colonialisme, suivi de Discours sur la Négritude, Paris, Présence africaine, 2000

(1950), 59 p.; Jacques Berque, Dépossession du monde, Paris, Éditions du Seuil, 1964, 215 p.

5. Mathieu Lapointe, «Entre nationalisme et socialisme : Raoul Roy (1914-1996) et les origines d'un premier indépendantisme socialiste au Québec, 1935-1965 », Mens, vol. 8, n² (printemps 2008), p. 281-322.

6. Andrée Fortin, loc. cit., p. 81-83.

7. Mathieu Lapointe, loc. cit., p. 300. 
américains. Mais le discours va plus loin : un véritable mythe de l'infériorité des Canadiens français s'est imposé dans la mémoire collective québécoise. La Conquête et les rébellions des Patriotes de 1837-1838 sont les évènements historiques les plus souvent rappelés à la société québécoise par les nationalistes qui, en soulignant comment la culture canadienne française y a survécu, en font des symboles de résistance à l'assimilation. Mais dans les faits, ces événements sont aussi grandement perçus comme l'illustration de l'échec, de l'infériorité et de la situation d'opprimés des Canadiens français ${ }^{8}$. L'émergence du « socialisme de décolonisation » s'inscrit dans ce contexte, et le climat de confrontation, voire d'accusation et de critique, va mener à la création, par Raoul Roy et ses collègues, de La Revue socialiste.

\section{CRItique des nationalistes}

Les nationalistes de droite sont les premiers à faire l'objet de critiques. Ils sont accusés de traîtrise pour s'être associés aux exploiteurs des Canadiens français, aux gouvernements collaborateurs de la colonisation et aux grands capitalistes:

Au Canada français, le nationalisme s'est identifié avec ceux qui ont voulu mobiliser le peuple pour le mettre au service d'un conservatisme immobiliste et de «l'entreprise privée» des étrangers. En somme, ce fut un écran de fumée pour masquer la complète reddition de notre petite bourgeoisie au capital dont la langue des propriétaires n'est pas la nôtre?

En plus de ces propos, Raoul Roy soutient que malgré l'évidence d'une lutte nationale, il n'était pas possible, dans ce contexte, à un homme de gauche, anticolonialiste, de se réclamer du socialisme. Ce type de vocabulaire aurait inévitablement été associé à la droite collaboratrice des régimes colonialiste ${ }^{10}$, ou bien aurait été considéré comme le propos de rêveurs croyant toujours à l'expansion de la culture française à la grandeur de l'Amérique du Nord ${ }^{1}$.

8. Catherine Bouchard, Les nations québécoises dans l'Action nationale : de la décolonisation à la mondialisation, Québec, Les Presses de l'Université Laval, 2002, p. 52-55.

9. Raoul Roy, «Le Québec est une colonie! Sus au colonialisme! », La revue socialiste, n 6 (1962), p. 8.

10. Ibid., p. 9.

11. Paul Moreau, « Procès de nos intellectuels », La revue socialiste, n² (automne 1959), p. 31. 


\section{Critique des « pSeudo-socialistes " et naissance du SOCIALISME DÉCOLONISATEUR}

Ceux qui sont toutefois les plus fortement accusés de trahison se retrouvent dans les courants de la gauche et de la "vieille gauche $»^{12}$. Les socialistes canadiens-anglais reconnaissent bien que les Canadiens français ont été un peuple particulièrement exploité économiquement dans I'histoire du prolétariat. Le fondateur de La Revue socialiste est d'ailleurs très proche de la gauche canadienne durant la plus grande partie de sa jeunesse ${ }^{13}$. Cependant, les partis socialistes réaffirment leur antinationalisme, d'où la trahison perçue par les intellectuels nationalistes de gauche du Québec. Ceux-ci les accusent d'occulter la dimension ethnique de l'exploitation et de travailler à la continuation plus subtile, mais plus efficace, de l'exploitation des Canadiens français en la diluant dans la « masse » des travailleurs à libérer ${ }^{14}$. L'article «Trahison du socialisme » de Raoul Roy ne peut être plus clair : "Que partout dans l'univers, les socialistes dignes de ce nom ne craignent pas d'être à l'avant-garde du combat des peuples coloniaux pour leur indépendance, excepté au Canada français $[. . .]^{15}$. »

Il parle en outre des partis socialistes comme des « pseudo-socialistes » qui, bien que prônant la «libération ouvrière », ne soutiennent pas pour autant la «libération nationale ». Or, pour Roy, il s'agit là des deux objectifs primordiaux qui devraient toujours se trouver au centre des combats menés par les «vrais socialistes ». D'ailleurs, puisque les partis socialistes demandent, selon lui, une centralisation économique appuyée d'une centralisation politique pour pouvoir mieux redistribuer les richesses, il les perçoit, dans leur objectif d'assimilation des Canadiens français, comme des suppôts de la bourgeoisie anglophone ${ }^{16}$. II considère aussi encore plus grande la trahison de certains de leurs membres qui, bien qu'issus du Canada français, ne s'expriment pas sur ce sujet. Les expériences personnelles de Raoul Roy dans les partis fédéraux de gauche suffisent sans doute à justifier certaines de ses critiques - il quitte d'ailleurs le Parti communiste du Canada lorsque des Canadiens français y sont accusés de " déviation nationaliste ${ }^{17}$. II est toutefois également possible de lier celles-ci et la pensée générale de Roy aux écrits d'autres intellectuels qui, à l'international, ont milité pour la décolonisation du Tiers-monde.

12. Mathieu Lapointe, loc. cit., p. 300.

13. Ibid., p. 294.

14. Ibid., p. 300.

15. Raoul Roy, «Trahison du socialisme », La revue socialiste, n² (automne 1959), p. 6.

16. Ibid., p. 1-7.

17. Mathieu Lapointe, loc. cit., p. 296. 
Parmi eux, Frantz Fanon est sans doute l'un des auteurs qui a le plus fortement inspiré La Revue socialiste. Celle-ci n'hésite pas à encenser son ouvrage Les damnés de la terre, à encourager sa diffusion et à en faire la publicité à travers ses pages. C'est peut-être d'ailleurs pourquoi le cas de l'Algérie semble revenir si souvent dans la revue. Plusieurs auteurs citent Fanon ou font référence à ses écrits pour formuler leurs critiques de la gauche. C'est le cas d'André Major, futur fondateur de la revue Parti pris, qui fera paraître dans La Revue socialiste un article s'attaquant à la « vieille gauche », c'est-à-dire les libéraux comme Pierre-Elliot Trudeau, et à son internationalisme " qui n'est qu'une mystification propre au régime colonial ${ }^{18}$. »

Dans le même numéro que "Trahison du socialisme », Roland Cousineau appuie quant à lui les propos de Raoul Roy par l'exemple algérien, en rappelant directement les articles de Frantz Fanon sur le « réformisme type Union française » adopté par le Parti communiste algérien malgré les ambitions indépendantistes des rebelles du F.L. ${ }^{19}$. Les rapprochements avec la gauche canadienne sont multiples et l'appui au F.L.N. est associé à une sorte de vieux sentiment anti-français plus qu'à un réel soutien aux mouvements de décolonisation en général.

\section{Le Québec Colonisé}

La province de Québec est-elle une colonie et, si tel est le cas, qui sont les « colonisateurs »? Ce questionnement va se trouver au cœur des écrits des intellectuels québécois défendant l'idéologie de la « décolonisation du Québec » à la fin des années 1950 et tout au long des années 1960. Non seulement ceux-ci tenteront-ils de répondre à ce questionnement, mais ils essayeront, bien souvent, de convaincre leurs lecteurs de les appuyer dans leur démarche indépendantiste. Les réponses formulées vont toutefois varier d'une revue à l'autre, d'un auteur à l'autre; sans réel consensus ${ }^{20}$.

\section{La VISION PolitiQue et ÉCONOMIQUe de La ReVUe socialiste}

Bien que les axes politiques et économiques ne soient pas les seules priorités des auteurs, il semble clair qu'il s'agit toutefois de l'un des points centraux de La Revue socialiste. Si les considérations économiques semblent rarement faire l'objet de débats au sein de la gauche québécoise, la réalité, selon l'intellectuel Albert Memmi, apparait fort différente à l'international. Selon lui, il aurait été difficile pour les Français, desquels les intellectuels québécois indépendantistes s'attendaient à recevoir du soutien, d'assimiler la situation sociale et éco-

18. André Major, « «les damnés de la terre» et nous », La revue socialiste, nº 6 (1962), p. 46.

19. Roland Cousineau, «L'Algérie et le Québec », La revue socialiste, n² (automne 1959), p. 41-44.

20. Sean Mills, op. cit., p. 5-6. 
nomique du Québec à celle de pays colonisés. En effet, si les Québécois ne bénéficiaient pas d'une richesse comparable à leurs voisins du continent américain, ils jouissaient pourtant d'une qualité de vie qui, du point de vue de l'Europe, demeurait enviable ${ }^{21}$.

Le manifeste politique de La Revue socialiste présente toutefois le Québec comme une société non seulement moins favorisée du point de vue économique, mais même comme sous-développée. La forte présence des intérêts anglo-canadiens et américains dans l'industrie québécoise est, à cet effet, mise en avant dans l'argumentaire des auteurs de la revue. Ils considèrent le Québec " occupé » économiquement, car les profits ne reviennent qu'aux étrangers qui, selon eux, profitent pourtant allègrement de la main-d'œuvre bon marché et des matières premières de la province. Ces points d'argumentation sont d'ailleurs présentés dans les « 100 points » du manifeste politique de la revue qui a été publié dans son premier numéro ${ }^{22}$. Outre ce manifeste, le directeur de la revue, Raoul Roy, renvoie ses lecteurs à un autre article pour connaître les raisons qui feraient du Québec une colonie ${ }^{23}$.

Dans un article publié à l'hiver 1959-1960 sous le titre "Québec, une sous-colonie », Roy explique toutefois de façon chronologique, en partant de la conquête de la Nouvelle-France, tous les évènements politiques majeurs qui, selon lui, auraient contribué à maintenir le Québec dans un statut de "colonie », voire même de «colonie d'une colonie ». À ses yeux, la province n'a jamais pu se doter de textes constitutionnels librement construits, ceux-ci lui auraient plutôt été imposés. En outre, il soutient que l'union des deux Canada était une décision de Londres et qu'elle a provoqué la perte de l'usage exclusif du nom de Canadiens aux francophones, une offense selon lui. La Confédération, ensuite, aurait été encore plus néfaste. Pour Roy, celle-ci a redéfini et, par le fait même, limité davantage les pouvoirs provinciaux. Elle aurait contribué à noyer la culture canadienne-française dans une majorité anglophone qui ne souhaitait céder à aucune égalité de droits.

II reprenait ainsi l'idée des théoriciens de la décolonisation stipulant que le colonisé, pour grimper l'échelle sociale et sortir de sa misérable condition, devait abandonner tout ce qu'il était afin de devenir un Canadien anglais ${ }^{24}$. II semblerait donc que l'assimilation, dans la pensée de Roy, ait été possible, voire même souhaitée par les Canadiens anglais. II soutient de plus l'idée selon laquelle les Québécois auraient jusqu'alors eu peu d'impact sur la gestion politique et économique de la province, et que cela aurait contribué, puisqu'ils auraient ainsi été privés d'une participation active à l'Histoire, à les plonger et à les maintenir dans une

21. Albert Memmi, op. cit., p. 138.

22. [s. a.], « Propositions programmatiques de la Revue socialiste », La revue socialiste, n 1 (printemps 1959), p. 13-14.

23. Raoul Roy, loc. cit., 1962, p. 5.

24. Raoul Roy, « Québec, une sous-colonie? », La revue socialiste, n 3 (hiver 1959-1960), p. 18-27. 
forme d'« enfance politique $»^{25}$. Cela rejoint en partie les propos d'Albert Memmi qui écrivait, dans son célèbre Portrait du colonisé, que le « fait est que le colonisé ne gouverne pas. Que strictement éloigné du pouvoir, il finit en effet par en perdre l'habitude et le goût ${ }^{26}$. »

Roy retourne aussi les critiques formulés par ses opposants fédéralistes concernant un prétendu désintéressement des Canadiens français envers le domaine politique; pour Roy, ils en sont eux-mêmes les fautifs ainsi que, de façon plus générale, le colonialisme. II en profite enfin pour rappeler que, comme ce serait le cas dans de nombreux pays colonisés - mais également selon ce que lui inspire sa vision plus traditionnelle de la culture canadienne-française -, c'est davantage à sa famille plutôt qu'à son pays que le Canadien français tend à se dévouer ${ }^{27}$. Ce phénomène, qui serait un symptôme de la colonisation, est d'ailleurs décrit par certains critiques du colonialisme. Memmi, par exemple, qualifie la famille et la religion comme des $«$ valeurs-refuges $»^{28}$.

Le Québec, selon Roy, n'a pas de pouvoir dans les domaines importants. Il s'emploie d'ailleurs à énumérer tous les pouvoirs fédéraux sur lesquels le gouvernement provincial n'a aucune influence et dont il dépend pourtant, tels la défense et la guerre (évoquant par le fait même les conscriptions forcées), l'organisation militaire, la douane, la Cour suprême, l'émission de la monnaie, la police fédérale, le droit de veto sur les lois provinciales et l'immigration, etc ${ }^{29}$. Son point de vue sur le danger que représente selon lui l'immigration du point de vue du maintien de la culture québécoise est par ailleurs l'un des principaux points de divergence avec les analyses présentées dans la revue Parti pris. En effet, les jeunes intellectuels de cette revue refusent d'être associés à une certaine forme de racisme ou de xénophobie. Ils vont à cet effet adopter une vision nationaliste davantage ancrée dans le domaine politique que dans le domaine culturel. De ce fait, leur vision de la nécessaire protection de la culture sera différente de celle de la Revue socialiste ${ }^{30}$.

\section{La vision sociale et culturelle de Parti pris}

Parti pris se voulait une vitrine d'expression pour les penseurs de la décolonisation au Québec et, ultimement, un outil d'accession à l'indépendance. C'est d'ailleurs dans cette logique que les auteurs souhaitant participer au projet général de cette revue se sont réunis et ont créé

25. Ibid., p. 30. Cet aspect est énoncé encore plus clairement dans André Major, loc. cit., p. 45.

26. Albert Memmi, op. cit., p. 114.

27. Raoul Roy, «Québec et la démocratie », La revue socialiste, n 1 (printemps 1959), p. 7.

28. Albert Memmi, op. cit., p. 117-119.

29. Raoul Roy, loc. cit., hiver 1959-1960, p. 18-20.

30. Mathieu Lapointe, op. cit., p. 303-304. 
un club visant la mobilisation de militants ${ }^{31}$. Les angles politiques et économiques présentaient donc une grande importance pour ceux qui ont contribué à la revue Parti pris, mais l'analyse culturelle demeurait également primordiale. En observant le seul index des articles, il est en effet possible de relever de nombreuses collaborations artistiques, notamment de courts poèmes, des chansons et des nouvelles à saveur politique. Les arts et leur développement font partie des thèmes régulièrement abordés, comme, également, la littérature, le cinéma et même l'architecture montréalaise. Le sport y a aussi sa place. L'idée, en somme, était de conscientiser la population sur la dénaturation de la culture québécoise par le colonialisme, puis d'utiliser les arts comme un outil supplémentaire pour parvenir, finalement, à l'indépendance nationale et politique ${ }^{32}$.

La décolonisation est notamment abordée dans trente-cinq articles différents. Dans ce lot, vingt-huit touchent directement le Québec, dont une chronique qui s'étend du mois de mai 1966 au mois de mai 1967. Les thèmes de la « dépossession de soi » et de l'« aliénation », qu'on retrouve généralement chez la plupart des critiques du colonialisme, reviennent sans cesse dans la revue ${ }^{33}$. L'aliénation, en particulier, est un concept primordial qui est défini par l'entremise de discours sur la décolonisation.

Elle est, pour certains, la raison grâce à laquelle le système colonial a pu perdurer durant plusieurs décennies, mais - du fait même de ses conséquences néfastes sur le colonisé c'est également elle qui a progressivement provoqué sa chute. C'est du moins la perception de l'auteur et psychiatre Frantz Fanon dans son ouvrage Peau noire, Masques blancs. II souhaitait d'ailleurs, par cet ouvrage, faire prendre conscience aux colonisés de leur « aliénation collective »; prise de conscience qu'il considérait comme la première étape vers une libération nationale ${ }^{34}$. «Condamné » à une catégorie sociale par les caractéristiques négatives qui lui sont attribuées et en lesquelles il finit lui-même par croire, le colonisé serait coincé dans son univers social et psychologique. Jacques Berque décrit d'ailleurs les peuples colonisés comme des «peuples opprimés ou supprimés, ou tout au moins niés », aux cultures « dégradées en qualité, appauvries quant à la quantité, devenues à la fois chose et fantôme ${ }^{35}$. » Un de ses articles, repris également dans le septième numéro de La revue socialiste, est publié dans Parti pris en décembre 1963. Selon l'auteur, les Québécois subissent une forme parti-

31. Stéphanie Angers et Gérard Fabre, Échanges intellectuels entre la France et le Québec (1930-2000). Les réseaux de la revue Esprit avec La relève, Cité libre, Parti pris et Possibles, Québec, PUL, coll. "Sociologie contemporaine », 2004, p. 125.

32. Pour consulter l'index de la revue, voir Joseph Bonenfant, dir., Index de «Partipris» (1963-1968), Sherbrooke, Centre d'étude des littératures d'expression française, 1975, 116 p.

33. Stéphanie Angers et Gérard Fabre, op. cit., p. 148-149.

34. Renate Zahar, L'œuvre de Frantz Fanon : colonialisme et aliénation dans l'œuvre de Frantz Fanon, Paris, Masрего, 1970, p. 39-43.

35. Jacques Berque, op. cit., p. 61. 
culière de colonisation, peut-être plus subtile, mais qui les ferait tout de même souffrir d'une certaine forme de « dépersonnalisation ». La question de l'identité du Québécois serait par ailleurs particulièrement troublante, faite d'exceptions : " canadien, oui, mais français, français, mais d'Amérique, américain, mais en victime plus qu'en bénéficiaire ${ }^{36}$. »

Un autre article, écrit par Laurent Girouard et paru également en décembre 1963, rappelle quant à lui les propos émis par Memmi en ce qui concerne l'écrivain colonisé : « Nous en sommes à la dernière «aliénation» si l'on continue à croire que la vie culturelle est possible pour un peuple minoritaire durant le processus de son assimilation linguistique. La culture n'est viable que pour un peuple libre. [...] L'homme isolé n'écrit pas ${ }^{37}$. » Tout en décrivant la pauvreté qui caractérise le monde de l'édition au Canada français, l'auteur y raconte l'isolement des écrivains eux-mêmes, qui sont «écartelés entre une Grande Culture Européenne et la Gigantesque civilisation Américaine ", tout en étant, selon lui, entourés d'un peuple sans culture parlant le joual plutôt que le français ${ }^{38}$. Le thème de la langue est d'ailleurs omniprésent dans la revue. Si le «mauvais parler » québécois - le joual - pouvait être vu comme un signe d'aliénation relevant d'une situation coloniale vécue, il pouvait aussi représenter, selon certaines figures artistiques importantes de Parti Pris, telles Gaston Miron, Paul Chamberland et Raoul Duguay, le meilleur outil pour exprimer (voire crier) son identité et sa condition ${ }^{39}$.

\section{UN AVANT-GOÛT DE LA FIN?}

Alors que l'itinéraire de La Revue socialiste ne semble pas avoir été marqué d'embûches internes particulièrement importantes, le parcours de Parti pris apparait fort différent. Fondée en 1963 par cinq jeunes universitaires (Paul Chamberland, Pierre Maheu, André Brochu, Jean-Marc Piotte et André Major), la revue, comme nous l'avons déjà souligné, a été grandement influencée par les auteurs de la décolonisation. Mais ses orientations idéologiques témoignent également d'un attachement marqué à des figures du marxisme, notamment Lénine et Gramsci, en particulier en ce qui a trait aux idées de la « libération », par le socialisme, de la nation québécoise et du peuple québécois. Or, tous les fondateurs et les collaborateurs de la revue ne semblaient pas accorder la même importance à ces deux formes de libération ${ }^{40}$. Qui plus est, les années 1965-1966 vont marquer une période de grands bouleversements dans la direction de la revue puisque quatre des principaux membres de la ré-

36. Jacques Berque, « Les révoltés du Québec », Parti pris, vol. 1, n 3 (décembre 1963), p. 49-50.

37. Laurent Girouard, « Notre littérature de colonie », Parti pris, vol. 1, n 3 (décembre 1963), p. 31.

38. Ibid., p. 33.

39. Raoul Duguay, "Gérald Godin ou du langage aliéné bourgeois au langage aliéné prolétaire », Parti pris, vol. 4, n 5-6 (février 1967), p. 95.

40. Stéphanie Angers et Gérard Fabre, Échanges intellectuels entre..., p. 123. 
daction (Major, Piotte, Chamberland et Maheu) vont peu à peu s'en éloigner suite au constat de l'échec de leurs actions politiques concrètes.

\section{SOCIALISME OU INDÉPENDANCE?}

Dans la revue Parti pris, une attention particulière est portée aux luttes révolutionnaires d'Amérique latine et aux mouvements contestataires américains comme les Black Panthers ${ }^{41}$. Les sujets couvrent donc l'actualité internationale, mais celle-ci est généralement reliée à la « situation coloniale » du Québec. Si, ainsi que le soutient Andrée Fortin, la perte de popularité de la gauche radicale québécoise de la fin des années 1960 jusqu'à la fin des années 1970 était en partie due à un « oubli » progressif de la lutte nationale au profit d'un socialisme plus international ${ }^{42}$, il semblerait que cela n'ait pas été les orientations poursuivies par Parti pris. Certains articles concernent davantage le monde international, mais le Québec n'est jamais marginalisé ou laissé de côté dans les analyses proposées par les collaborateurs à la revue ${ }^{43}$. En outre, l'actualité québécoise sert généralement de fil conducteur et alimente à la fois les prises de position et les désaccords entre les différents auteurs qui participent à Partis pris.

À titre d'exemple, les événements de 1967-1968 - formation du Mouvement souveraineté-association et, par la suite, du Parti québécois - vont polariser les opinions des collaborateurs de la revue quant à la priorité des luttes. Bien que les lecteurs de Parti pris demandent à ce qu'on leur « montre le chemin » à suivre, les auteurs n'arrivent pas à mettre en œuvre un consensus et une ligne de pensée unanime face au contexte politique et social québécois $^{44}$. C'est par là même que le rédacteur Gabriel Gagnon explique devoir mettre un terme à la revue qui, selon lui, se montre incapable de survivre aux mouvements sociaux « qu'elle a contribué à susciter ${ }^{45}$. » Le " socialisme de décolonisation » semble donc avoir porté en lui une cause importante de son déclin.

\section{LA PRÉdICTION ET LA PERSÉVÉRANCE de RAOUL ROY}

Les problèmes rencontrés par la revue Parti pris nous poussent à rappeler les mises en garde exprimées par Raoul Roy à l'égard du marxisme dans la quête de la souveraineté nationale. En 1965, dans le dernier article de La Revue socialiste, intitulé « Socialisme, décolonisation et nationalisme », Roy avait choisi de mettre en scène trois personnages représentant chacun

41. Ibid., p. 124-127.

42. Andrée Fortin, Ibid., p. 81-83.

43. Les index de la revue en témoignent d'ailleurs. Voir Joseph Bonenfant, dir., op. cit..

44. [s.a.] « Parti pris, le R.I.N. et le M.S.A », Parti pris, vol. 5, nº 7 (avril 1968), p. 5.

45. Stéphanie Angers et Gérard Fabre, op. cit., p. 127. 
une idéologie, à savoir le marxisme, le nationalisme et le « socialisme de décolonisation $»^{46}$. Chacun d'eux tenait un discours sur la marche à suivre pour libérer le peuple québécois. Au terme de l'échange, Roy affirmait clairement que la première révolution à faire devait être nationale et que, s'il le fallait, le peuple devait s'allier à la partie des classes dominantes qui était prête à le suivre.

Celle-ci, selon Roy, ne devait pas être considérée, comme le voudrait un point de vue marxiste, comme un seul bloc ennemi ${ }^{47}$. Par ailleurs, Roy n'hésitera pas à critiquer les revues Parti pris et Révolution québécoise (créée par Pierre Vallières et Charles Gagnon suite à leur collaboration dans Parti pris ${ }^{48}$ ) quant à leurs positions et à leur critique de «l'impérialisme américain ». Il ira d'ailleurs jusqu'à demander aux collaborateurs de ces deux revues de taire un peu leurs opinions antiaméricaines et leurs élans vers la " guérilla de la révolution planétaire », un objectif que Roy jugeait trop élevé pour un peuple qui n'était toujours pas souverain et solide sur ses propres fondements ${ }^{49}$. Peut-être pouvons-nous voir, dans les inquiétudes formulées par Roy et par la perte de vitesse de la revue Parti pris, une certaine forme de déclin de la gauche radicale et du « socialisme de décolonisation »?

\section{Conclusion}

La recherche par certains intellectuels, à la fin des années 1950 et tout au long des années 1960, des responsables d'un « retard » des Canadiens français révèle leur perception de la situation sociale et politique du Québec. Peuple colonisé, les Canadiens français, aux yeux de la gauche radicale, avaient souffert de la trahison des nationalistes traditionnels et des socialistes antinationalistes. Cette représentation mènera au développement d'un nouveau courant idéologique directement inspiré du contexte international, à savoir le « socialisme de décolonisation ». La Revue socialiste sera au centre de ce courant et s'emploiera à adapter les théories de la décolonisation à la situation sociopolitique du Québec. En traitant d'enjeux politiques et économiques, elle se distinguera de la revue Parti pris qui accordera une importance particulière au domaine culturel et qui se tournera également davantage vers une vision internationale du « socialisme de décolonisation ».

Les revues intellectuelles de la gauche radicale ne disparaitront pas après l'arrêt de parution de La Revue socialiste et de Parti pris. En outre, de nombreux évènements, telles les actions du FLQ et la victoire du Parti québécois aux élections de 1976, entre autres, tendent à démontrer que le discours développé dans ces revues a continué d'évoluer malgré leur disparition

46. Raoul Roy, "Socialisme, décolonisation et nationalisme », La Revue socialiste, n 8 (1965), p. 23-24.

47. Ibid., p. 31.

48. Stéphanie Angers et Gérard Fabre, op. cit., p. 121.

49. Mathieu Lapointe, op. cit., p. 307-309. 
et s'est affirmé à l'intérieur de situations concrètes de la scène sociopolitique québécoise. L'analyse de ces deux revues est donc importante pour comprendre la récupération et l'adaptation des discours internationaux par certains intellectuels québécois des années 1960, puis le développement d'une « prise de parole » qui contribuera sans aucun doute au développement d'une idéologie dont les finalités prévoyaient la libération nationale et politique du Québec et des Québécois. 


\section{SOURCES ET BIBLIOGRAPHIE}

[s. a.]. «Propositions programmatiques de la Revue socialiste ». La revue socialiste, no 1 (printemps 1959), p. 13-33.

[s. a.]. « Parti pris, le R.I.N. et le M.S.A». Parti pris, vol. 5, nº 7 (avril 1968), p. 3-6.

ANGERS Stéphanie et Gérard FABRE. Échanges intellectuels entre la France et le Québec (1930-200). Les réseaux de la revue Esprit avec La relève, Cité libre, Parti pris et Possibles. Sainte-Foy, Les Presses de l'Université Laval, 2004, 248 p.

BALTHAZAR, Louis. Bilan du nationalisme au Québec. Montréal, L’Hexagone, 1986, 212 p.

BERQUE, Jacques. «Les révoltés du Québec ». Parti pris, vol. 1, n 3 (décembre 1963), p. 48-51.

BERQUE, Jacques. Dépossession du monde. Paris, Éditions du Seuil, 1964, 215 p.

BONENFANT, Joseph, dir. Index de «Parti pris » (1963-1968). Sherbrooke, Centre d'étude des littératures d'expression française, 1975, 116 p.

BOUCHARD, Catherine. Les nations québécoises dans l'Action nationale: de la décolonisation à la mondialisation. Québec, Presses de l'Université Laval, 2002, 140 p.

CÉSAIRE, Aimé. Discours sur le colonialisme, suivi de Discours sur la Négritude. Paris, Présence africaine, 2000 (1950), $59 \mathrm{p}$.

COUSINEAU, Roland. «L’Algérie et le Québec ». La revue socialiste, n² (automne 1959), p. 4-44.

DUGUAY, Raoul. «Gérald Godin ou du langage aliéné bourgeois au langage aliéné prolétaire ». Parti pris, vol. 4, nº 5-6 (février 1967).

FANON, Frantz. Les damnés de la terre. Paris, La Découverte, coll. "La Découverte/Poche », n 134, 2002 (1961), $311 \mathrm{p}$.

FORTIN, Andrée. «Les revues de gauche au Québec, 1960-1970 ». Bulletin d’histoire politique, vol. 19, $\mathrm{n}^{\circ} 2$ (hiver 2011), p. 78-86.

GIROUARD, Laurent. «Notre littérature de colonie ». Parti pris, vol. 1, n³ (décembre 1963), p. 30-37.

LAPOINTE, Mathieu. «Entre nationalisme et socialisme: Raoul Roy (1914-1996) et les origines d'un premier indépendantisme socialiste au Québec, 1935-1965 ». Mens, vol. 8, n² 2 (printemps 2008), p. 281-322.

MEMMI, Albert. Portrait du colonisé : précédé de portrait du colonisateur. Montréal, L'Étincelle, 1972 (1957), $146 \mathrm{p}$.

MAJOR, André. «"Les damnés de la terre" et nous ». La revue socialiste, n 6 (1962), p. 45-47.

MILLS, Sean. The Empire Within. Postcolonial Thought and Political Activism in Sixties Montreal. Montréal, McGill-Queen's University Press, 2010, 302 p. 
MOREAU, Paul. «Procès de nos intellectuels ». La revue socialiste, n² (automne 1959), p. 31-32.

ROY, Raoul. « Québec et la démocratie ». La revue socialiste, nº 1 (printemps 1959).

ROY, Raoul. «Trahison du socialisme ». La revue socialiste, n² (automne 1959), p. 1-8.

ROY, Raoul. «Québec, une sous-colonie? ». La revue socialiste, n³ (hiver 1959-1960), p. 17-61.

ROY, Raoul. «Le Québec est une colonie! Sus au colonialisme! ». La revue socialiste, nº (1962), p. 5-18.

ROY, Raoul. «Socialisme, décolonisation et nationalisme ». La revue socialiste, nº 8 (1965), p. 23-48.

ZAHAR, Renate. L'œuvre de Frantz Fanon : colonialisme et aliénation dans l'œuvre de Frantz Fanon. Paris, Maspero, 1970, 116 p. 